\section{A FORTRAN program for providing feedback to individual subjects from a standard case by variable data matrix}

\section{DAVID W. GERBING \\ Michigan State University, East Lansing, Michigan 48824}

Within the last decade there has been increasing concern over the treatment of human subjects as participants in psychological studies. One aspect of this concern has been the developing obligation on the part of the experimenter to provide feedback to the participants. It is in this context that the following feedback program was written.

Description. The program was initially written to provide feedback to respondents of Likert-type questionnaires. However, the program works for any data matrix in which each record (or set of records) consists of an identification number followed by the responses of an individual or some other other unit of analysis to a set of items or variables. The data format is provided by the user.

The user selects the items and the order in which they are printed by organizing the items into subscales. Any subset of items may be selected in any order and items may be listed in as many subscales as desired. Designated items are reflected and missing data are permitted, with the restriction that missing data are coded as single numbers that apply to the entire data matrix. Across individuals, the program computes the mean for each selected item and for each defined scale, as well as the minimum and maximum response to each scale. The response of an individual to each selected item is identified and his or her composite score for each scale is computed. In addition, the individual's $\mathrm{z}$ scores on selected items and on the scales are computed at the user's option. Individuals with an identification field equal to zero are dropped from the analysis of individual responses. To facilitate the distribution of the printed feedback forms to the respondents, an option is provided to sort the cases by the identification number. Since only one case at a time is read by the program, relatively little central memory is required by the execution of the program.

Input. Input consists of two data files. One file is the data matrix, in which the data for each case consists of a record or set of records. The default allows the identification number to precede the item responses, but an option is provided to reverse this order. The identification number may be as large as desired and may be split into several sections, since it is read-in digit by digit by a user-supplied format.

The second input file consists of the instructions. The user supplies the title, the integer format of the identification field and the item responses, the total number of items to be read, the number of scales, the number of digits in the identification number, and the number of items on each user-defined scale. After specifying the use of any options, the user lists, on separate records, the title of each scale followed by the items that define the scale. Reflected items are prefaced by a negative sign.

Output. The program first prints various bookkeeping information, such as the data format, the default conditions, the activated options, the items identifying each scale, and the reflected items. If the missing data option has been selected, the number of nonmissing elements for each item is also printed.

The bulk of the output consists of the feedback forms sorted by the identification number. On all but very large sets of items, each form should consist of a single page of computer output labeled with the title and identification number. To conserve paper, the scale titles, the individual and group item responses and composite scores, and, if desired, $z$ scores of the items and scales are printed across the page in sets of five scales. The output of each set of five scales begins on the same line even though the scales may be of varying length. Thus it is possible to print the results of, for example, up to 15 scales, with a maximum of 10 items per scale on each page. If the single space option is selected over the default value of double spacing, more scales may be printed on a single page. Missing data are printed as - 99 .

Computer and Language. The program is written in FORTRAN for use on the CDC 6500 . Although some $\mathrm{CDC}$-specific code has been incorporated in the program by necessity, standard ANSI code was used whenever possible. Thus, conversion to other versions of FORTRAN should be relatively simple.

Limitations. The maximum number of items is currently 200 , and the item responses are in integer format. However, minor changes to DIMENSION and FORMAT statements would remove these restrictions.

Availability. A source listing, instructions, and sample input and output are available at no cost from the author, Department of Psychology, Michigan State University, East Lansing, Michigan 48824.

(Accepted for publication November 29, 1978.) 